\title{
Comment « pragmatiser » le champ de la valeur?
}

How to make the field of value more pragmatic?

\section{Louis Quéré}

\section{OpenEdition}

\section{Journals}

Édition électronique

URL : http://journals.openedition.org/questionsdecommunication/11502

DOI : 10.4000/questionsdecommunication. 11502

ISSN : 2259-8901

\section{Éditeur}

Presses universitaires de Lorraine

\section{Édition imprimée}

Date de publication : 31 décembre 2017

Pagination : 195-218

ISBN : 9782814305076

ISSN : 1633-5961

\section{Référence électronique}

Louis Quéré, «Comment «pragmatiser » le champ de la valeur? », Questions de communication [En ligne], 32 | 2017, mis en ligne le 31 décembre 2019, consulté le 04 janvier 2021. URL : http:// journals.openedition.org/questionsdecommunication/11502; DOI : https://doi.org/10.4000/ questionsdecommunication.11502 
LOUIS QUÉRÉ

Institut Marcel Mauss

Centre national de la recherche scientifique

École des hautes études en sciences sociales

F-750006

louis.quere@ehess.fr

\title{
COMMENT «PRAGMATISER » LE CHAMP DE LA VALEUR ?
}

\begin{abstract}
Résumé. - Cette contribution discute les propositions faites par Nathalie Heinich pour développer une sociologie des valeurs. Elle en critique certaines - l'anti-naturalisme, par exemple, le refus de considérer la religion comme une référence pertinente pour caractériser les valeurs, la séparation de l'éthique du champ de la valeur, la limitation de ce dernier aux attachements, actes évaluatifs et jugements de valeur, etc. Elle tente d'en clarifier d'autres - le lien des valeurs aux affects et aux émotions, le statut des « valeursprincipes », le caractère ultime des valeurs, le statut des explicitations, etc. La contribution adopte le point de vue méthodologique recommandé par John Dewey pour analyser le champ de la valeur : s'en tenir à une approche adverbiale, c'est-à-dire considérer les valeurs comme manières de faire et comme instruments de la conduite. Un tel point de vue permet de pousser plus loin le tournant pragmatique préconisé par Nathalie Heinich, qui s'inspire plutôt de la pragmatique linguistique.
\end{abstract}

Mots clés. - affects, autorité, conviction morale, émotions, évaluations, idéaux, incertitude, jugement, motricité, partage, principes, réactions esthétiques, standards, « valuations » 
L es clarifications proposées par Nathalie Heinich (2017a, 2017b) dans sa sociologie des valeurs sont précieuses, notamment sur la non-réductibilité de la valeur au prix ou à ce que mesurent les sondages d'opinion, sur les différentes significations du mot « valeur », sur la pluralité des valeurs et des modes de qualification, de même que le sont ses propositions pour une approche pragmatique, soucieuse de saisir les modalités et les contextes des opérations d'évaluation, ou encore sa défense d'un « relativisme descriptif ». Mais sa description de « l'expérience axiologique » me paraît quelque peu biaisée faute de saisir son envergure, son ancrage naturel, sa composante émotionnelle et sa dimension pratique, et, finalement, de sortir du paradigme « représentationnaliste » dominant (voir sa conception des valeurs comme « représentations mentales partagées »).

En fait, ce que je propose est que l'on pousse jusqu'au bout la « pragmatisation » du champ de la valeur, ce qui suppose que l'on ne lie pas l'ontologie de la valeur uniquement aux « attachements », aux actes et opérations d'évaluation, ou à la formation de jugements de valeur énonçables discursivement. Pour ce faire, mon point d'appui sera la théorie pragmatiste de la « valuation » de John Dewey, qui repose sur une distinction (servant essentiellement à des fins de clarification conceptuelle) entre « valuations » et « évaluations 》. Mais je suis d'accord avec le refus de Nathalie Heinich d'une sorte d'interdit sociologique concernant les valeurs : ce n'est pas parce que l'invocation répétée des valeurs dans le discours public est le plus souvent une défense (de droite) des traditions, des institutions et des mœurs établies (qui sont des manières socialement « valuées 》 de se conduire), que le sociologue doit se garder de parler de valeurs et d'en comprendre la dynamique et la fonctionnalité. Ce dont il doit plutôt se garder est de commettre l'équivalent, en sociologie, du « sophisme du psychologue » (James) ou du « sophisme du philosophe » (Dewey) - c'est-à-dire d'« introduire dans l'expérience première, comme faisant partie de sa nature immédiate, tout ce qu'une analyse postérieure peut y trouver » (Dewey, 1934a :215). Il peut le faire en expliquant, en termes de conduites et de manières d'agir, de moyens et de modes d'interaction avec l'environnement, à quoi correspondent exactement ces valeurs si souvent invoquées dans le discours social'.

\section{La valeur comme manière d'agir}

Nathalie Heinich propose à juste titre d'échapper à l'essentialisme concernant les valeurs; elle s'applique à le faire en considérant les valeurs comme des représentations mentales, individuelles et collectives, et en faisant porter l'enquête sociologique principalement sur les actes et les opérations d'évaluation et de justification. Mais il faut d'abord mieux comprendre le geste dont procède cet essentialisme récurrent.

' Certains passages de cet article sont repris du texte d'un exposé fait à l'EHESS et disponible sur le site du CEMS dans la rubrique Occasional Papers (http://cems.ehess.fr/docannexe/file/37/4/op33_lq_ autorite_des_valeurs.pdf). 
Pour l'essentiel, il consiste à transformer en une substance ce qui n'a, de fait, qu'une réalité fonctionnelle ou modale. Celui qui fait de la valeur une catégorie spécifique de choses existantes, désignée par des noms abstraits, commet un sophisme de ce type. À propos de l'esprit, de la matière et de la conscience, John Dewey écrivait en 1925 :

« On peut prédire de façon plausible que si on nous interdisait pendant toute une génération d'utiliser les mots "esprit", "matière", "conscience" comme noms et qu'on nous obligeait à employer des adjectifs et des adverbes, "conscient" et "consciemment", "mental" et "mentalement", "matériellement" et "physiquement", un grand nombre de nos problèmes en serait considérablement simplifié » (Dewey, 1925 : 97, trad. mod.).

Deux décennies plus tard, il faisait la même recommandation méthodologique au sujet de la valeur, terme qu'il conseillait de traiter comme un adjectif, pour éviter les pièges de l'usage de noms abstraits (Dewey, 1949). En fait les noms abstraits ne font que récapituler, en résumé, des qualités de l'expérience.

Un adjectif caractérise habituellement un nom. Mais un adverbe est aussi un adjectif, un adjectif du verbe précisément : il spécifie la manière d'agir indiquée par ce dernier. Adopter une approche adverbiale des valeurs c'est les considérer d'abord comme des manières d'agir situées, impliquant des qualifications anté-prédicatives, observables dans les attitudes et les comportements qu'un organisme adopte dans ses transactions avec son environnement. Dans ces transactions, il « value » en permanence des objets, des événements, des situations, des comportements, des pratiques, des relations, y compris le déroulé de ses propres accomplissements, ainsi que leurs fins et leurs moyens, leurs résultats et leurs conséquences. Ces « valuations » sont fonctionnelles, au sens où elles contribuent à diriger les changements constitutifs de la conduite.

II n'est peut-être pas inutile de préciser trois points liés à cette approche adverbiale. D'abord, qu'un environnement n'est pas simplement un ensemble de ressources et de contraintes (comme on le pense souvent en sociologie) mais aussi un « co-opérateur »- les opérations à travers lesquelles nos conduites et nos activités prennent forme sont distribuées entre l'organisme et son environnement (ce que désigne le terme «transaction »). Ensuite, que la conduite (conduct) est une affaire d'orientation et de contrôle de changements dans une activité qui est un procès séquentiel et sériel, changements qui sont plus ou moins coordonnés avec ceux qui se produisent concomitamment dans l'environnement (Dewey, 1930). Enfin, que les qualifications effectuées dans un jugement transforment en objets de pensée des totalités qualitatives directement éprouvées dans l'expérience immédiate (Dewey, 1931).

En fait « tout ce qui se trouve sous le soleil » peut être « valué », c'est-à-dire qualifiéen-valeur. Mais les qualifications-en-valeur excèdent largement les évaluations proprement dites ou les jugements de valeur; elles ne créent donc pas nécessairement des « valeurs-objets » ou des « biens » (quand je « value » les moyens de mes actions, je n'en fais pas des « valeurs-objets »). Les qualifications-en-valeur sont pour une grande part purement pratiques, comportementales et situationnelles ; 
elles ont néanmoins un visage ou une physionomie, qui permet de les identifier ; elles suscitent aussi des expressions : ce que l'on est enclin à dire ou disposé à faire dans leur sillage. On dispose de toute une série de verbes pour décrire ce mode de qualification : donner du prix, de la valeur ou de l'importance à, tenir à, chérir, estimer, priser, apprécier, admirer, honorer, approuver, révérer, donner du poids à, défendre, être dévoué à, porter de l'intérêt à, se préoccuper de, etc. Tous ces verbes sont positifs, ce qui traduit la connotation positive des termes « valeur » et « valuer ». Mais on doit aussi tenir compte de la bivalence des qualifications-envaleur : des verbes tels que dédaigner, décrier, déprécier, détester, mésestimer, haïr, mépriser, vomir, soupçonner, etc., expriment des qualifications négatives.

Les termes « valuer », « valuation » n'existent pas en français (on ne peut pas utiliser « valorisation », qui a un autre sens), mais il me semble nécessaire d'utiliser ces anglicismes pour tenir compte du fait que les évaluations proprement dites et lesdits « jugements de valeur » ne représentent qu'une des modalités des qualifications-en-valeur, et aussi du fait que les comportements attestant des «valuations » ont une source biologique et une dimension affective-motrice. Comme Nathalie Heinich refuse de considérer les valeurs comme des faits de nature, je vais discuter son point de vue. Par ailleurs, si elle relève bien la pluralité des modes de qualification, dans lesquels elle inclut les « attachements », elle privilégie néanmoins les actes évaluatifs et les opérations d'évaluation soustendant les jugements - ce qui, à mes yeux, réduit excessivement le champ de la valeur et risque d'introduire un biais cognitiviste dans l'analyse (oubli de l'ancrage de la cognition - pensée, réflexion, jugements, etc. - dans une expérience immédiate irréfléchie).

II n'y a d'ailleurs pas que les valeurs et les modes de qualification qui sont pluriels : les régimes de valeur le sont aussi. II y a des régimes scientifiques, religieux, philosophiques, moraux, esthétiques de la « valuation ». Si l'on prend le cas de ce dernier, force est de convenir qu'il n'est pas réservé au domaine de l'art. Une activité ordinaire peut avoir une valeur esthétique, comme peuvent l'avoir un échange ou une conversation (voir l'exemple de l'entretien d'embauche dans le chapitre 3 de L'Art comme expérience de John Dewey), ou donner lieu à des « valuations » de nature esthétique. Lorsque j'utilise un marteau pour enfoncer des clous dans une planche, je peux apprécier plusieurs choses dans l'expérience que j'en aie : le caractère adapté à ma main de la forme et de la taille du manche, source de confort, son adéquation à la masse en métal, sa parfaite convenance pour la tâche à effectuer (il aurait pu être trop léger ou trop lourd ; le matériau dont il est fait aurait pu ne pas s'avérer approprié pour la tâche à effectuer ; par exemple, s'il avait été en bois ou en caoutchouc, quelque chose m'aurait gêné, m'empêchant d'apprécier), etc. L'expérience elle-même est « valuée » dans son développement, de même que l'activité dans laquelle j'utilise mon marteau : positivement, si elle se déroule sans encombre, si les opérations s'enchaînent de manière fluide et si le résultat satisfait - la « valuation » est alors de nature esthétique - ; négativement, si tout va de travers, si l'activité est hachée, désordonnée ou éclatée, ou si le résultat déçoit. 
LudwigWittgenstein (cité in Chauviré, 2004 : 53) parlait de « réactions esthétiques 》 pour désigner de telles « valuations » spontanées. « La chose la plus importante en rapport avec l'esthétique est peut-être ce que l'on peut appeler les réactions esthétiques $\gg$. Un exemple de réaction esthétique est la non-satisfaction ou l'inconfort devant un objet - devant le dessin d'une porte que l'on a fait par exemple : trop haute, trop basse, mal proportionnée par rapport à la façade, aux fenêtres. Quand je réagis ainsi au dessin, il s'agit d'une « valuation » affective-motrice immédiate. Je peux cependant formuler cette réaction, par exemple en disant : « elle est trop haute ».Je produis alors une évaluation critique de mon dessin en même temps que j'exprime ma non-satisfaction. Je pourrais aussi l'exprimer par une moue particulière ou un geste. Si l'expression n'est pas nécessaire au fait que je ressente une non-satisfaction ou une gêne, toutefois, lorsque je ne suis pas satisfait par quelque chose, de mon dessin de la porte par exemple, je suis enclin à dire, à faire telle ou telle chose. Pour Ludwig Wittgenstein les réactions esthétiques sont dans la continuité des réactions naturelles, c'est-à-dire des réactions qui ne procèdent pas de la pensée, du calcul ou de l'interprétation, mais appartiennent « aux faits généraux de la nature » (si nous n'avions pas réagi « par nature » au rythme d'une certaine manière, nous n'aurions pas eu la musique et les danses que nous avons). II s'agit d'un mode de conduite pré-linguistique, mais qui peut être formulé et affiné à l'aide du langage.

À la différence des évaluations, les « valuations » ne sont pas un type d'acte spécifique et elles ne reposent pas sur des opérations. Elles font partie du cours normal d'une conduite et sont incorporées, comme moyens d'orientation et de contrôle, dans ce que l'on fait et subit. Elles sont purement pratiques et se manifestent d'abord dans des modes de comportement. Par conséquent, une sociologie qui se focalise plutôt sur les actes évaluatifs et les jugements dits « de valeur » risque de passer à côté d'une part importante des « valuations », d'autant plus qu'elle oublie aussi souvent que les jugements de valeur, quand ils sont des jugements authentiques, c'est-à-dire quand ils procèdent d'un examen réfléchi, et ne sont pas simplement des réactions immédiates ou l'expression d'impressions premières, sont avant tout des jugements de pratique : ils servent à diriger les changements constitutifs de la conduite, par la formation de désirs et par l'anticipation de conséquences probables, et ils guident les « valuations » subséquentes.

Cette dernière notation est importante : les jugements de valeur influencent, voire changent, les «valuations » immédiates. Je ne partage donc pas le point de vue de Nathalie Heinich (2017b : 312) selon lequel « la sociologie des valeurs [...] n'a rien à voir avec une quelconque éthique ». On ne peut soutenir une telle position qu'en oubliant que les jugements de valeur sont des jugements de pratique, c'est-à-dire qu'lls concernent l'organisation de la conduite, et notamment la détermination des fins à poursuivre - ils donnent leur direction aux activités et contrôlent leur accomplissement - et que souvent ils réorientent les « valuations » immédiates. Dans cette organisation, les valeurs issues de ces évaluations sont tour à tour des fins et des moyens. Elles sont notamment des moyens pour appréhender les conséquences, et donc pour définir de nouvelles fins. Généralement, les fins et les moyens sont eux-mêmes « valués ». 


\section{Sur le refus du naturalisme}

Les valeurs, nous explique Nathalie Heinich, sont des « faits de culture et non pas de nature ». J'ai plutôt envie de dire qu'ils sont les deux à la fois, et que le refus d'un certain naturalisme n'est pas justifié. II peut se comprendre parce que le naturalisme est souvent réductionniste - par exemple quand il fait du cerveau et des hormones l'assise de la vie sociale. Mais, s'agissant de « valuations », force est de convenir qu'elles ont une base naturelle biologique, c'est-à-dire qu'elles font partie du comportement instinctif des êtres vivants dans leurs interactions avec leur environnement. En effet, pour assurer leur survie, ils procèdent constamment à des sélections et à des rejets, sur la base d'attractions et de répulsions ; ils vont vers ce qui les attire, cherchent à l'obtenir, et évitent, voire éliminent, ce qui ne convient pas ou leur déplaît ; ils adoptent ainsi des comportements ou des attitudes qui manifestent qu'ils « valuent » positivement ou négativement les éléments de leur environnement. Parmi ces comportements figurent, du moins pour les animaux évolués, ceux qui consistent à prendre soin de leurs rejetons, à les nourrir et à les protéger, parce qu'ils leur accordent de la valeur, voire à adopter des comportements d'empathie vis-à-vis de leurs congénères (comme le suggèrent certaines études récentes en éthologie).

Comme le souligne cette description, ces comportements instinctifs ont une dimension affective (aimer, ne pas aimer; apprécier, détester, etc.) et une dimension motrice (aller vers, favoriser, s'approcher de ; s'écarter de, empêcher, se garder de, éliminer, se défendre). La dimension motrice est importante. Elle correspond à la fois à l'effectuation de mouvements et à la dépendance des sensations par rapport à des activités motrices. Quand nous savourons quelque chose, un mets par exemple, notre jouissance repose sur une interaction sensori-motrice avec l'objet apprécié, interaction qui est un mélange d'activités (ce que nous faisons dans notre bouche avec la langue, le palais, etc.) et de passivités (ce que nous subissons).

Cependant, il manque aux comportements purement instinctifs l'élément intellectuel qui leur conférerait une dimension de désir. Celui-ci apparait quand l'être vivant inscrit ses « valuations 》 affectives-motrices dans une visée de résultats pour ses accomplissements. Soit l'exemple de John Dewey : une poule qui viserait consciemment, comme fin ou résultat de son activité, l'éclosion des œufs qu'elle couve ajouterait une telle dimension à la « valuation » à la fois des œufs, dont elle prend soin, et de son activité, qu'elle accomplit avec application. Elle serait porteuse d'un désir. La « valuation » affective-motrice deviendrait alors une « valuation » à la fois « affective, idéationnelle et motrice » (Dewey, 1938 : I I5).

Les « valuations » affectives-motrices ne disparaissent pas chez l'homme, mais elles se complexifient en incorporant et développant un tel élément intellectuel ; cette incorporation permet qu'elles se transforment non seulement en « désirs », mais aussi en évaluations proprement dites, y compris en évaluations d'elles-mêmes, à toutes fins pratiques s'entend (par exemple, méritent-elles d'être maintenues comme bases d'action ?). Une « valuation » immédiate peut ainsi s'avérer à la 
réflexion indésirable ou problématique ; son évaluation réflexive la transforme de même qu'elle transforme l'objet « valué ». II y a ainsi à la fois une continuité et une discontinuité entre les «valuations » affectives-motrices immédiates et les évaluations proprement dites, dont font partie les jugements de valeur : « Bref, le jugement de valeur est le développement délibéré d'un constituant aspectuel des prizings et cherishings plus directs, dans lesquels doivent continuellement s'engager les êtres humains en tant que créatures vivantes, et sous des conditions telles qu'ils sont d'abord relativement "irréfléchis" ("thoughtless")² 》 (Dewey, 1949: 354).

Ce développement délibéré n'est cependant qu'une des formes possibles de l'évaluation. Celle-ci peut aussi n'être que l'expression d'une impression première ; il lui manque alors la solidité qu'elle a lorsqu'elle est la conclusion d'un examen réfléchi, étayé sur une perception approfondie. De toute façon, toutes les évaluations, dont les jugements de valeur, ont pour point de départ des impressions de nature qualitative produites en nous par les choses et les événements (ceux-ci sont d'abord « sentis »). Notons qu'une évaluation en bonne et due forme, dépassant la réaction immédiate, ne cherche pas nécessairement à attribuer une valeur à un objet : elle ne se préoccupe pas tant d'estimer l'objet considéré que d'en approfondir la perception, en saisissant ses propriétés objectives; cette appréhension ne conduit pas nécessairement à un jugement sur la valeur d'ensemble de l'objet, mais elle peut le faire.

On peut donc naturaliser sans problème le champ de la valeur. Mais cette naturalisation relèvera d'un naturalisme culturel, car les humains sont des êtres acculturés. Leur « expérience axiologique » est, comme toute expérience, une affaire d'interactions immédiates avec un environnement, interactions où se mêlent et s'équilibrent le subi et l'agi. Ces interactions sont réglées par des impulsions et des habitudes. C'est cette intervention des habitudes, qui canalisent les impulsions, qui fait que l'arrière-plan des « valuations » affectives-motrices immédiates est en grande partie social et culturel. En effet, l'acquisition d'habitudes inclut aussi bien la formation de coordinations sensori-motrices que celle d'attitudes émotionnelles et intellectuelles ; elle façonne notamment notre affectivité instinctive tout comme nos réactions diverses et variées à ce qui nous entoure ou à ce qui nous arrive. Ces habitudes sont formées dans un milieu social-historique ; elles sont inculquées dès le plus jeune âge par des comportements adoptés par les autres dans l'environnement social - approbation/désapprobation, récompense/punition, injonction/interdit, etc. Ce sont, par ce biais, les mœurs, les us et coutumes, les institutions en vigueur et les préjugés qui conditionnent (causalement, peut-on dire) les « valuations » affectives-motrices immédiates, sans compter toutes sortes de manipulations possibles de la part des détenteurs du pouvoir économique, politique ou culturel. L'analyse de ce qui conditionne ainsi les « valuations » et les évaluations, ainsi que celle de leurs conséquences, sont des tâches de la sociologie des valeurs (à supposer que cette spécialité académique existe).

\footnotetext{
2 « Judgment of values, in short, is the deliberate development of an aspectual constituent of the more direct prizings and cherishings that human beings as living creatures must and do continually engage in, and under such conditions that at first they are relatively "thoughtless" ».
} 
Nathalie Heinich parle d'« expérience axiologique ». Si elle entend par là un type spécifique d'expérience, je crois qu'elle se fourvoie : ce que je viens d'expliquer sur l'omniprésence des « valuations » dans les conduites et les activités tendrait à prouver que la dimension « axiologique » est plutôt une composante inévitable de toute expérience, plutôt qu'un type spécifique d'expérience.

\section{Les émotions comme sondes des valeurs}

La composante émotionnelle des « valuations 》 (on les a dites « affectivesmotrices, ou affectives-idéationnelles-motrices ») et des évaluations est essentielle. Les émotions entrent inévitablement en jeu dans les qualifications-envaleur, essentiellement parce que « le monde de notre vie immédiate, celui dans lequel nous combattons, réussissons et sommes vaincus, est avant tout un monde qualitatif. Ce pour quoi nous agissons et souffrons, ce à quoi nous prenons plaisir ce sont les choses dans leurs déterminations qualitatives ${ }^{3} \gg$ (Dewey, |931 : 93). Ces déterminations qualitatives sont « senties », d'où l'importance des affects. II suffit, pour s'en convaincre, de penser à nombre de qualificatifs-en-valeur qui sont des termes émotionnels, dénotant des émotions caractéristiques - qu'il s'agisse de qualificatifs apparemment neutres (bon, beau, agréable, honnête, charitable, cruel, etc.) ou de qualificatifs exprimant plus explicitement des affects (émouvant, exaltant, dégoûtant, répugnant, gênant, horrible, effroyable, etc.).

C'est par nos émotions que nous nous rendons compte de ce qui nous est cher, de ce qui nous importe, de ce à quoi nous tenons, de ce à quoi nous sommes attachés... ou encore du contenu réel de nos orientations du moment, de nos préoccupations, de nos préférences, de nos objectifs ou de nos attentes. II en va ainsi parce que nos émotions sont suscitées par un différentiel entre ce qui advient, d'un côté, nos orientations, préférences, attentes, valeurs, etc., de l'autre. Dans les termes de Pierre Livet (2002, passim), les émotions sont « les sondes de nos valeurs ». Et, le plus souvent, c'est en partageant nos émotions que nous sommes assurés du caractère commun et partagé de nos repères collectifs, dont font partie lesdites valeurs. Toutefois, au-delà de cette fonction de révélation, les émotions font aussi un véritable travail. Ce sont elles qui opèrent les sélections et les rejets évoqués supra.

L'idée de «travail » des émotions n'est pas absente de la psychologie et de la sociologie. Ainsi, en psychologie, dans l'approche des émotions en termes d'« appraisal », le travail de l'émotion est-il défini en termes d'appréciation et d'évaluation immédiates des situations, et de production d'ajustements rapides. Les personnes évaluent émotionnellement ce qui arrive et ce à quoi elles sont confrontées sous l'aspect de ses implications pour leur bien-être personnel, et y répondent avant toute réflexion. En sociologie, l'idée de «travail émotionnel » a

\footnotetext{
3 "The world in which we immediately live, that in which we strive, succeed, and are defeated is preeminently
} a qualitative world. What we act for, suffer, and enjoy are things in their qualitative determinations ». 
été introduite par Arlie Hochschild (1979), dans une perspective goffmanienne de gestion de la « face ». Mais on peut aller plus loin et considérer que le travail de l'émotion ne consiste pas seulement à apprécier et évaluer les situations, et à susciter des réponses rapides, mais aussi à sélectionner les éléments pertinents d'une situation pour la traiter, et à unifier le divers de l'expérience, notamment en reliant ses différentes composantes (intellectuelle, pratique, affective).

\section{Les valeurs sont-elles des principes?}

Une des significations que Nathalie Heinich confère au mot « valeur » est celle de « principe d'évaluation » : la « beauté », la « responsabilité », la « moralité », l'《 authenticité », la « fidélité », la « générosité », l'《 efficacité », la « décence », etc. pourraient être considérées comme de telles « valeurs-principes ». Celles-ci fonctionneraient comme «causes » des évaluations. Elles auraient pour propriétés d'être « autotéliques », c'est-à-dire de « ne reposer sur rien d'autre qu'ellesmêmes », d'être « à elles-mêmes leur propre but et leur propre nécessité », et de mettre fin «à la série des "pourquoi" justificateurs de l'action » (Heinich, $2017 b$ : 299). Cette caractérisation est très intéressante, mais elle mérite d'être discutée. Prenons le cas de la « beauté ». La beauté est à la fois une modalité de qualification-en-valeur, un critère d'évaluation et, sans doute, une forme d'idéal. Est-elle aussi un principe doté d'un pouvoir causal ? J'en doute fortement. Pour justifier mon scepticisme, je vais considérer plusieurs points.

On a souvent noté le risque d'hypostase que comporte l'usage d'un terme abstrait tel que « beauté » (mais ce n'est pas parce que le terme est abstrait que la valeur désignée l'est aussi) : à partir de l'extase émotionnelle (il s'agit d'une émotion particulière) provoquée par la capacité d'un objet de susciter l'admiration, la beauté acquiert, par une hypostase, le statut d'une substance pure, voire celui « d'une essence offerte à l'intuition » (Dewey, 1934a : I 62). Pour éviter l'erreur inhérente à une telle conversion d'un terme émotionnel en une substance ou une essence, à laquelle est encline toute restitution intellectualisée d'une expérience immédiate qui est d'abord qualitative, il vaut mieux partir de l'adjectif («c'est beau ! ») qui exprime une émotion. Et lorsqu'on utilise le terme « beauté » pour désigner « la qualité esthétique globale d'une expérience », il est recommandé de « s'attacher à l'expérience ellemême » et de comprendre d'où cette qualité procède et comment; dans ce cas, « la beauté est la réponse à ce que représente, pour la réflexion, le processus par lequel le mouvement accompli par un matériau est intégré par ses relations internes dans une totalité qualitative unique » (ibid. ; je souligne).

A priori, un terme émotionnel ne dénote pas un principe d'évaluation. Mais qu'est au juste un tel principe ? On peut élucider quelque peu ces questions en distinguant standards, principes et critères. Certaines évaluations relèvent de la mesure ; elles requièrent alors des standards ou des étalons de mesure. Un standard peut être considéré indépendamment de la fonction qu'il exerce dans 
une évaluation. II en va ainsi, par exemple, pour le nuancier Mansell utilisé par les archéologues pour différencier les couleurs du sol qu'ils explorent. II comporte de nombreux échantillons de couleur. En un sens, ces échantillons ne sont jamais que du papier imprimé en couleur et on peut dire un certain nombre de choses sur leurs différences et leurs similitudes. Mais lorsqu'ils sont rapprochés des couleurs du sol que l'archéologue veut déterminer, ils acquièrent une valeur d'étalons de mesure et deviennent un outil pour la qualification et la justification. II nous faut ainsi des standards, c'est-à-dire des invariants extérieurs et publics, fixés conventionnellement, pour pouvoir identifier les choses, les qualifier, les différencier, faire des comparaisons, etc. Mais ils ne sont des outils que lorsqu'ils sont en opération active dans une pratique de mesure. En réalité les standards sont moins des valeurs que des règles (bien qu'ils puissent avoir une valeur), et la principale opération sous-tendant leur usage est la comparaison. Une « valeurprincipe » telle que la beauté ne semble pas de cet ordre, car elle n'est pas suffisamment déterminée et elle n'est pas vraiment prescriptive.

À vrai dire, tous les standards ne sont pas des règles. Ils peuvent aussi incarner des idéaux de référence assez flous. Les différentes pratiques (en artisanat, médecine, sciences et techniques, arts, etc.) comportent des standards d'excellence qui leur sont inhérents ; s'y engager c'est déférer à ces standards, qui deviennent alors non seulement des critères d'évaluation de ses propres performances et de celles des autres, mais aussi des repères pour développer ses compétences et orienter l'exercice de ses capacités (Mclntyre, 198I). Ces standards sont cependant différents des règles parce qu'ils ne sont pas prescriptifs et que les idéaux qu'ils incarnent sont peu déterminés.

Ce n'est pas le genre de médiation fourni par les standards ou les étalons de mesure qui semble impliqué dans les jugements de valeur. Certaines évaluations sont des mesures, mais en général juger n'est pas mesurer. Mesurer c'est comparer une chose à une autre pour déterminer une valeur ou attribuer un prix (tel objet mesure telle longueur, pèse tant, a tel volume, telle couleur, vaut tant, etc.) pour faire une opération déterminée, tandis que juger requiert d'appréhender les qualités d'un objet individuel, à travers une interaction avec lui, qui peut être plus ou moins approfondie ; il faut que l'objet pénètre l'expérience de celui qui juge « au travers de sa sensibilité, ses connaissances et tout ce qu'il a emmagasiné de ses expériences passées » (Dewey, 1934a : 357). Le recours à une règle extérieure et publique prédéterminée ne peut y avoir qu'une place tout à fait subordonnée. Certes une appréciation est faite eu égard à des valeurs, mais les qualités appréciées ne sont pas « catégorisées en tant que valeurs » (ibid. : 356).

Le jugement requiert des critères, plutôt que des standards et des étalons de mesure ; les critères ne sont pas extérieurs et ils ne sont aucunement prescriptifs; ils sont des repères pour l'appréciation qualitative d'un objet sous différents aspects. Pour apprécier l'objet il faut le percevoir d'une certaine façon ou sous un aspect - par exemple, sous l'aspect de sa composition, de sa structure, de sa cohérence, de son homogénéité, de son harmonie, de l'assemblage de ses 
couleurs, de l'intégration de ses parties dans le tout, de sa congruence avec ce qui l'environne, etc. Celui-ci qui est affligé d'une « cécité à l'aspect » (incapacité à saisir une forme ou une totalité organisée dans un ensemble, ou les traits pertinents d'une composition) ne parviendra pas à apprécier qualitativement un objet. En général, l'appréciation qualitative est multi-critérielle. Elle requiert aussi des capacités et des techniques, bref un savoir-faire (exemples : savoir déguster un vin, apprécier une performance musicale, etc.).

Maintenant, les critères de jugement sont-ils des principes ?Tout dépend de ce qu'on entend par « principe ». On invoque des principes en logique, en mathématiques, en théorie de l'enquête, en philosophie morale et dans les discussions ordinaires sur le bien-fondé d'une conduite. Dans la tradition philosophique, on a toujours eu tendance à envisager les principes comme des choses rigides fixées a priori et extérieures aux processus d'évaluation eux-mêmes. Les pragmatistes ont critiqué cette propension et envisagé les principes plutôt comme des habitudes, des règles de méthode ou des hypothèses. En logique, les principes énoncent des habitudes d'inférence, qui produisent des conclusions stables dans les raisonnements (Peirce). Dans la théorie de l'enquête, ils formulent des opérations à exécuter, ainsi que des repères pour évaluer la conduite de l'enquête de l'intérieur de son accomplissement (Dewey). En morale, les principes sont des généralisations probabilistes et révisables, sans composante normative, dont nous nous servons dans nos jugements moraux. Ils formulent des façons de répondre à un certain type de situation, mais c'est de ce qu'impose la situation qu'ils tirent leur autorité et non pas d'une quelconque nature intrinsèque. En fait il s'agit le plus souvent de généralisations à partir d'enquêtes empiriques passées effectuées dans des situations problématiques. Ces généralisations fournissent des points de vue et des hypothèses pour délibérer en situation. En tant qu'hypothèses, les principes sont testés, confirmés, modifiés à partir des résultats et conséquences (« valués » et évalués) qui se produisent quand on les utilise comme base d'action.

Les valeurs satisfont-elles cette définition? En partie oui, et c'est ainsi que nous pouvons nous assurer de leur objectivité, c'est-à-dire en les prenant comme bases d'action et en voyant si nous « valuons » les conséquences qui en résultent: « Essayez et vous verrez si vous apprécierez vraiment. Si c'est le cas, vous pourrez vous y fier à l'avenir ». En mettant les jugements de valeur en pratique on obtient les données permettant de tester leur validité.

Les valeurs peuvent donc bien être traitées comme des principes. Mais il me semble qu'elles correspondent plutôt à des « conceptions du désirable », pour reprendre la définition de Talcott Parsons (1968: 136)4. Mais, en tant que telles, elles ne désignent pas des choses qui seraient en général ou a priori. Le désirable est pour une part élaboré dans ce que Charles Taylor (1989) appelle des « évaluations fortes », pour une autre part dans les projections de l'imagination idéalisatrice. Une « évaluation forte » tient compte des conditions réelles, telles qu'appréhendées

\footnotetext{
${ }^{4}$ «I therefore accept the first part of Kluckhohn's well-known definition of values as conceptions of the desirable ».
} 
dans le traitement des situations. Mais la détermination du « désirable » requiert aussi de pouvoir faire des distinctions entre ce qui mérite d'être maintenu ou écarté dans les « valuations » spontanées, entre ce qui mérite d'être poursuivi ou abandonné, et donc des distinctions entre le bien et le mal, le mieux et le pire, le supérieur et l'inférieur, etc. De telles distinctions supposent de pouvoir se référer à des critères indépendants des désirs, inclinations et choix du moment.

Ludwig Wittgenstein notait qu'on a tendance à sublimer les standards, à les désirer dans leur forme pure, débarrassée de leur matérialité. Mais c'est une illusion, car c'est grâce à leur matérialité que les étalons, du moins quand ils sont des choses physiques, sont des moyens de mesure et de représentation : « II ne peut y avoir de longueur sans un corps - et même si je comprends que, dans un sens déterminé, il n'y a que la longueur de la règle qui mesure, ce que je mets dans ma poche n'en reste pas moins la règle, le corps, et n'est pas la longueur » (Wittgenstein cité in Bouveresse, 2006 : 63). D'une certaine manière il en va de même pour les « valeurs-principes ». Dans ses Remarques sur le Rameau d'or de Frazer, Ludwig Wittgenstein note au sujet de la mort et de la beauté : " "Rien n'est aussi mort que la mort ; rien n'est aussi beau que la beauté elle-même". L'image sous laquelle on se représente ici la réalité consiste à penser que la beauté, la mort, etc. sont les substances pures (concentrées), alors qu'elles sont présentes comme ingrédient dans un objet beau » (in ibid. : 64). Cette propension à hypostasier des termes adjectivaux, à forte teneur émotionnelle, est évidemment très présente dans la conception que l'on a spontanément des valeurs.

On l'a dit, les valeurs sont le plus souvent désignées par des noms abstraits : beauté, vérité, justice, démocratie, égalité, liberté, solidarité, dignité de la personne, etc. On peut considérer à juste titre que de tels concepts ont introduit dans la vie de nouvelles valeurs, de nouvelles motivations, de nouveaux intérêts et de nouvelles incitations, et aussi de nouveaux sentiments d'admiration. William James (1909:224) demandait ainsi de ne pas oublier que ces concepts sont toujours à relier à la vie active : « Les gens qui possèdent certains concepts sont animés d'une certaine manière, ils poursuivent leur propre chemin vital différemment. II ne s'ensuit pas nécessairement qu'ils comprennent plus intimement les autres chemins vitaux ». William James notait aussi que « l'abstraction per se semble avoir une touche d'idéalité » et susciter une vénération que ne suscitent pas les réalisations concrètes des idées abstraites, qui apparaissent comme des parents très pauvres comparées à elles :

«La véritable valeur en espèces (cash-value) de l'idée semble ne s'attacher qu'à son statut abstrait. La vérité en général, comme Royce le soutient, [...] semble être tout à fait autre chose que les particuliers vrais auxquels il est bon de croire. Sa valeur transcende tous ces "expédients" ; elle est quelque chose pour lequel on doit vivre, expédient ou non. La Vérité avec un grand $V$ est une "question d'une importance capitale"; les vérités de détail ne sont que de "paurres bribes", de simples "miettes de succès" » (ibid.).

Bref, les idées abstraites et les noms abstraits suscitent une véritable vénération en raison de la « touche d'idéalité » qu'ils véhiculent, c'est-à-dire parce qu'ils désignent des idéaux, produits par l'imagination idéalisatrice. Mais, on le verra, la vénération n'est qu'une des attitudes que suscite un idéal. 
Une valeur « ne repose-t-elle sur rien d'autre qu'elle-même » (Heinich, 20 I 7b : 304) ? Représente-t-elle « une butée de l'argumentation » (ibid.), au sens où elle se suffirait à elle-même? Le risque, avec de telles formulations, est de donner crédit à ceux qui font des valeurs des choses ultimes, suprêmes, absolues. Pour éviter ce risque, il est d'abord conseillé de s'en tenir au seul sens temporel de termes tels que « ultime », « dernier », etc., comme l'ont suggéré Charles $\mathrm{S}$. Peirce et John Dewey :

«Quelque chose peut être ultime en ce sens qu'il vient en dernier dans une série temporelle, de telle sorte qu'il est ultime pour cette série. Il y a des choses qui viennent en dernier dans les "valuations" réflexives, et elles sont ultimes en tant que terminales. [...] Pour moi, la méthode de l'action intelligente est précisément une valeur ultime. Elle est la dernière chose, la chose finale ou dernière à laquelle nous arrivons dans une enquête sur l'enquête. Mais c'est la place qu'elle occupe dans la manifestation temporelle de l'enquête qui en fait une telle valeur, et non pas une propriété qu'elle possède en soi et par elle-même dans l'isolement de ce qui n'est pas mis en relation. Elle est ultime dans l'usage et la fonction ; elle ne prétend pas être ultime en raison d'une "nature inhérente" absolue, qui la rend sacrosainte, ou en fait un objet d'adoration transcendant ${ }^{5}$ » (Dewey, 1939: 77).

Une valeur peut donc être ultime moins en tant que «butée de l'argumentation » (on va le voir les valeurs ne font pas bon ménage avec l'argumentation) qu'en tant que point final de l'enquête.

Une autre stratégie pour éviter de faire des valeurs des choses ultimes, est de les rapporter non seulement à des contextes, mais aussi à des situations. On peut dire des valeurs ce que John Dewey disait des biens : elles sont toujours des valeurs d'une situation. Ainsi n'y a-t-il pas de bien en soi ou de bien absolu, mais uniquement des « biens d'une situation », découverts, lorsqu'il y a un conflit à résoudre entre des orientations ou des choix, par une réflexion sur des conditions et des conséquences : «Personne ne vit dans un monde dans lequel il trouve que tout est parfait à tout moment. S'il comprend la signification de ce fait, il a appris à être sensible à des possibilités. Le mieux potentiel sera alors considéré comme le bien - et le seul bien - de n'importe quelle situation » (Dewey, ibid. : 72). Ces biens/valeurs découverts dans la situation par la réflexion procèdent en fait souvent d'une réévaluation des biens/valeurs surgis dans l'expérience immédiate à travers des valuations affectives-motrices. II en va de même pour les critères : plutôt que d'être a priori et fixés indépendamment des pratiques, ils sont élaborés et configurés comme moyens par leur usage en situation.

Une fois qu'on s'est garanti contre le risque précédent, on peut effectivement dire qu'une valeur « ne repose sur rien d'autre qu'elle-même ». Mais cela suppose de

\footnotetext{
${ }^{5}$ « A thing may be ultimate in the sense of coming last in a given temporal series; so that it is ultimate for that series. [...] For me the method of intelligent action is precisely such an ultimate value. It is the last, the final or closing, thing we come upon in inquiry into inquiry. But the place it occupies in the temporal manifestation of inquiry is what makes it such a value, not some property it possesses in and of itself, in the isolation of non-relatedness. It is ultimate in use and function; it does not claim to be ultimate because of an absolute "inherent nature" making it sacrosanct, a transcendent object of worship ».
} 
considérer les valeurs plus comme des idéaux de référence, somme toute assez vagues, que comme des principes. Les idéaux ne « causent » absolument pas les comportements ; ils suscitent des convictions et des aspirations, des allégeances et des engagements; ils indiquent des directions pour la conduite, ou pour l'amélioration de la qualité de l'expérience, et ils rendent certaines expériences possibles... à condition, toutefois, qu'ils soient autre chose que des images de « substances pures ».

\section{Les valeurs comme idéaux}

Considérer les valeurs comme des idéaux, c'est déjà ce que faisait Émile Durkheim. En effet, pour lui les valeurs sont essentiellement des fins idéales, opérant comme forces de liaison et de motivation dans la vie sociale. C'est dans les expériences collectives d'effervescence créatrice, de nature émotionnelle, où la vie collective « atteint un certain degré d'intensité », que, selon Émile Durkheim, les idéaux se forment. La formation d'idéaux est, écrit-il, « une caractéristique essentielle des religions » (Durkheim, 1912 : 602). Elle a lieu dans des rituels qui sont des moments extraordinaires d'exaltation de la vie morale. La société y prend conscience d'elle-même et entretient le sentiment qu'elle a d'elle-même. Les idéaux sont ainsi des « produits naturels de la vie sociale » (ibid. : 603). Nathalie Heinich (2017b : 294) n'est pas favorable à ce rapprochement des valeurs, en tant qu'idéaux, de la religion : « Le paradigme religieux est sans pertinence concernant la question des valeurs ». En fait tout dépend de la conception que l'on se fait du religieux. II y a, me semble-t-il, une conception différente de celle d'Émile Durkheim qui peut éclairer la nature des valeurs.

Pour Émile Durkheim (1912:602), un idéal est rajouté au réel et mis à part, comme l'est le sacré : il « substitue au monde de la réalité un monde différent où [l'homme] se transporte par la pensée ». Surajouté au monde réel, l'idéal permet de s'élever au-dessus de l'expérience ordinaire et de concevoir un monde différent. C'est pourquoi, la création d'idéaux est vitale pour une société : « Une société ne peut ni se créer ni se recréer sans [...] créer de l'idéal. Cette création n'est pas pour elle une sorte d'acte surérogatoire, par lequel elle se compléterait, une fois formée ; c'est l'acte par lequel elle se fait et se refait périodiquement » (ibid.).

Pour Émile Durkheim (1970 : 265), les valeurs ont une autorité, qui provient pour une grande part de leur sacralisation, qui les met « en dehors et au-dessus de tous les intérêts temporels » et suscite crainte et respect à leur égard, ainsi qu'amour et attirance (parce qu'elles idéalisent et dépeignent une vie nouvelle).

L'individu ou la personne humaine représente une telle valeur dans les sociétés modernes : la dignité de l'individu est devenue la « religion de l'humanité », et l'individu est devenu l'objet d'un culte dont il est « à la fois l'objet et l'agent » : 
«Cette personne humaine, dont la définition est comme la pierre de touche d'après laquelle le bien se doit distinguer du mal, est considérée comme sacrée, au sens rituel du mot pour ainsi dire. Elle a quelque chose de cette majesté transcendante que les Églises de tous les temps prêtent à leurs Dieux; on la conçoit comme investie de cette propriété mystérieuse qui fait le vide autour des choses saintes, qui les soustrait aux contacts vulgaires et les retire de la circulation commune. Et c'est précisément de là que vient le respect dont elle est l'objet. Quiconque attente à une vie d'homme, à la liberté d'un homme, à l'honneur d'un homme, nous inspire un sentiment d'horreur, de tout point analogue à celui qu'éprouve le croyant qui voit profaner son idole. Une telle morale n'est donc pas simplement une discipline hygiénique ou une sage économie de l'existence ; c'est une religion dont l'homme est, à la fois, le fidèle et le Dieu » (Durkheim, 1970 : 264-65).

Et Émile Durkheim d'expliquer comment l'homme est devenu un dieu pour l'homme, et « la religion de l'humanité » la religion d'aujourd'hui. Cette nouvelle religion a tous les attributs classiques de la religion : la personne humaine est la seule chose à laquelle peut « désormais se prendre la sensibilité collective » (ibid. : 27I) et assurer « la communion des esprits » :

«Dès qu'une fin est poursuivie par tout un peuple, elle acquiert, par suite de cette adhésion unanime, une sorte de suprématie morale qui l'élève bien au-dessus des fins privées et lui donne ainsi un caractère religieux. D'un autre côté, il est évident qu'une société ne peut être cohérente s'il n'existe entre ses membres une certaine communauté intellectuelle et morale » (ibid. : 270-7I).

Hans Joas a consacré un livre à l'histoire de cette sacralisation de la personne et à la généalogie des droits de l'homme. La genèse des valeurs est, écrit-il, un processus contingent, et « nos valeurs sont des individualités historiques ${ }^{6}$ » (Joas, 2013 : 3). Elles ne peuvent orienter les conduites que si elles sont maintenues par des institutions et incorporées dans des pratiques. Ce qu'il faut surtout expliquer c'est comment se forment nos engagements en faveur de valeurs (valuecommitments) : «Notre engagement en faveur de valeurs et notre conception de ce qui a de la valeur émergent de nos expériences et de la manière dont nous les traitons ; c'est pourquoi ils sont contingents plutôt que nécessaires ${ }^{7}$ » (ibid.).

Une des caractéristiques des engagements en faveur des valeurs est, souligne Hans Joas, qu'ils ont une composante affective. C'est ce qui fait que nous avons des valeurs autrement que nous avons des opinions ou des croyances; ce n'est pas sur la base d'une reconnaissance de leur validité cognitive que nous y adhérons (ce que laisse trop sous-entendre le critère de la « butée de l'argumentation » retenu par Nathalie Heinich). Leur pouvoir de conviction n'est pas celui de la persuasion discursive par des arguments, mais celui, spécifique, d'un idéal. Par conséquent la communication au sujet de valeurs ne peut pas se réduire à un échange d'arguments rationnels. Hans Joas rappelle notamment qu'on ne peut pas discuter de valeurs de « façon atomistique », comme s'il s'agissait d'opinions discrètes, autonomes ; elles forment des groupes ou des grappes où elles sont entremêlées.

\footnotetext{
${ }^{6}$ « Our values are historical individualities 》.

7 « Our commitment to values and our notion of what is valuable emerge from experiences and our processing of them; this shows them to be contingent rather than necessary ».
} 
À la différence d'Émile Durkheim, Hans Joas ne considère pas la sacralisation de la personne humaine comme un phénomène de même nature que la religion. Néanmoins, dans la mesure où elle est de l'ordre d'un idéal, elle a quelque chose à voir avec le religieux. C'est un point de vue que l'on trouvait déjà chez John Dewey, notamment dans son essai sur le religieux, intitulé Une foi commune ( 1934b). Pour John Dewey, le religieux connote non pas l'adhésion aux croyances et pratiques d'une religion instituée, mais une attitude pratique, qui se concrétise par le choix d'un mode de vie sur la base d'une allégeance à des idéaux : « Je devrais définir la foi religieuse comme l'unification du soi par l'allégeance à des fins idéales inclusives, que l'imagination nous présente et auxquelles réagit la volonté humaine en les considérant comme dignes de présider à nos désirs et à nos choix » (Dewey, 1934b : 24). Cette allégeance a bien une composante affective, mais elle est essentiellement une orientation pratique, imposant des exigences et impliquant de s'efforcer de se changer soi-même et de changer le monde par ses pratiques. Elle relève de la conviction morale, qui est d'une autre nature que l'assentiment intellectuel à des croyances ou à des arguments :

« Avoir une conviction au sens moral signifie être conquis, vaincu, par une finalité idéale, et ce, dans notre nature active. Cela signifie reconnaître cette finalité dans sa demande légitime à exercer son primat sur nos désirs et nos buts. Une telle reconnaissance est pratique et non pas principalement intellectuelle. [...] L'autorité d'un idéal qui s'impose à nos choix et à notre conduite est l'autorité d'un idéal : ce n'est ni l'autorité d'un fait, ni l'autorité d'une vérité garantie à l'intellect, ni l'autorité qui découlerait du statut de celui qui proclame une soi-disant vérité » (ibid. : I06).

Les attitudes constitutives du religieux n'ont donc rien d'extraordinaire ni d'irrationnel ; elles appartiennent à la dynamique de l'expérience naturelle des humains, à savoir à celle de la formation et de l'unification, par l'imagination créatrice, de fins idéales ou d'idéaux dotés d'autorité, bref de valeurs. Ces valeurs sont extraites des associations humaines par la saisie de possibilités réelles dans l'expérience : il s'agit de projections à partir des biens dont on fait concrètement l'expérience dans les relations familiales, dans les relations de voisinage et de travail, dans l'exercice de la citoyenneté, dans la pratique des arts ou dans la science. Mais on a toujours eu tendance à les substantialiser et on a constamment voulu adosser leur autorité à celle d'un être surnaturel ou à des forces invisibles. On a hypostasié les fins idéales projetées et unifiées par l'imagination dans une existence extérieure et antécédente. Mais les idéaux n'ont pas besoin d'être soutenus par de telles forces, car ils ne sont pas en rupture avec l'expérience ordinaire ; ils n'ont pas besoin d'un autre support que les habitudes, les pratiques et les significations qui animent celles-ci :

« J'ai essayé de montrer que l'idéal s'enracine dans les conditions naturelles et émerge lorsque l'imagination idéalise l'existence en saisissant les possibilités offertes à la pensée et à l'action. II est des valeurs, il y a du bien, qui sont effectivement réalisés sur une base naturelle : le bien des associations humaines, de l'art et de la connaissance. L'imagination idéalisatrice se saisit des choses les plus précieuses qu'elle trouve dans les moments privilégiés de l'existence et les projette. Nous n'avons pas besoin de critères extérieurs pour garantir leur teneur en bien. On les a, elles existent comme bonnes, et à partir d'elles, nous forgeons nos fins idéales » (ibid. : I37- I38). 
Et ce n'est pas parce qu'ils sont produits par l'imagination que les idéaux sont faits de « matériaux imaginaires » : « Ils sont en dur, de la matière dont est fait le monde de l'expérience dans ses aspects physiques et sociaux » (ibid. : I39).

Ces idéaux servent d'outils d'évaluation, de moyens de direction de la conduite et de bases pour la critique : « Ils fournissent les significations à partir desquelles la vie est jugée, estimée et critiquée » (Dewey, 1925 : 193). Ils permettent notamment d'évaluer et de critiquer ce qui existe, et de rendre sensible ce qui manque. II en est ainsi parce qu'ils génèrent « un sens des possibilités » qui fait découvrir des aspects négatifs de la réalité :

«Un sens des possibilités qui ne sont pas réalisées, mais qui pourraient l'être, et qui, lorsqu'elles sont mises en contraste avec les conditions réelles, sont la "critique" la plus pénétrante qui puisse être faite de ces dernières ; c'est par un sens des possibilités s'offrant à nous que nous prenons conscience des constrictions qui nous enserrent et des poids qui nous oppressent » (Dewey, 1934a : 396).

On n'est pas ici dans le registre de la causalité.

John Dewey précise cependant que toute foi morale dans des finalités idéales n'est pas pour autant religieuse. II faut que cette foi produise, grâce à l'émotion, une unification du self et son harmonisation avec l'univers:

« Le religieux n'est "la moralité touchée par l'émotion" que lorsque les fins de la conviction morale suscitent des émotions non seulement intenses mais aussi déclenchées et soutenues par des fins si inclusives qu'elles unifient le soi. Le caractère inclusif de cette fin en rapport avec le soi et l'“univers" - auquel est lié un soi inclusif - est indispensable » (ibid. : 108).

En un sens, John Dewey rejoint ainsi Émile Durkheim dans sa définition des valeurs en termes d'idéaux, dotés d'une forme spécifique d'autorité, de nature morale. Mais comme, avec George H. Mead, il rejette l'idée que la société serait un être psychique particulier « d'un genre nouveau » (Émile Durkheim) - la société n'est pas mentale ; l'esprit, l'imagination, la conscience ne sont pas le lieu de la société, martèle George H. Mead -, comme, par ailleurs, il se refuse à relier d'emblée le religieux au sacré ou au surnaturel, et qu'il récuse l'explication de l'organisation de la vie sociale par des forces collectives sui generis (notamment des représentations collectives), il peut être plus économe et plus empiriste qu'Émile Durkheim dans l'analyse de la genèse sociale des valeurs : celles-ci correspondent aux projections de l'imagination idéalisatrice, reprenant des possibilités découvertes dans le réel. C'est donc l'expérience elle-même qui dévoile les valeurs, et à leur tour celles-ci rendent possibles de nouvelles expériences : « La valeur de l'expérience ne réside pas seulement dans les idéaux qu'elle révèle, mais dans son pouvoir de dévoiler divers idéaux [...]. On peut même renverser l'affirmation et dire que la valeur des idéaux réside dans les expériences qu'ils rendent possibles » (Dewey, 1934a : 370).

À l'origine de l'allégeance que les valeurs suscitent il y a donc non la sacralisation des idéaux, comme chez Émile Durkheim, ni la capture de l'affect commun généré par l'imitation affective (comme chez Spinoza, repris parAndré Orléan [20 I I]), mais, d'un côté, l'attachement implicite, par conviction morale, à des fins idéales découvertes 
dans l'expérience, de l'autre, les émotions partagées suscitées par l'exploration, dans l'art, dans la communication et dans l'enquête sociale, de possibilités réelles non encore réalisées. Les émotions sont importantes, mais ce ne sont pas les émotions qui sont la source des valeurs et de leur autorité : ce sont l'imagination et l'ancrage dans la vie réelle des possibilités que l'imagination découvre et projette. Ce sont les fins idéales de la conviction morale qui suscitent des émotions, et non l'inverse.

\section{L'autorité des valeurs}

On a souvent rappelé que les valeurs ne sont pas universalisables comme les normes. II en irait ainsi parce qu'elles ne seraient que des préférences subjectives, éventuellement des préférences culturelles collectives (celle pour la liberté, l'égalité et la fraternité dans une République, par exemple), ou encore des biens désirés par tous. Si les valeurs ne sont que « des préférences intersubjectivement partagées 》 (Habermas, 1992 : 278), et si les jugements de valeur ne font qu'établir de telles préférences, en pondérant plusieurs critères, alors la question de la légitimité et de l'autorité des valeurs ne se pose pas; l'autorité est remplacée par la simple attraction de biens, dont on pense en commun qu'ils méritent qu'on s'efforce ensemble de les atteindre. On peut concéder que les valeurs ne puissent pas être considérées comme universelles ; mais si elles s'imposent comme choses dernières au terme de l'enquête (comme « la méthode de l'intelligence » dans la citation de John Dewey supra), elles sont en un sens universalisables.

On l'a vu, il existe quelque chose comme une allégeance aux valeurs qui est autre chose qu'une attraction pour des préférences : cette allégeance est de l'ordre de la soumission à une autorité ; ce qui suppose que les valeurs puissent s'imposer comme des choses légitimes et valant pour tous. Dans L'Empire de la valeur, André Orléan (20 I I) explique cette autorité par la captation de la « puissance de la multitude », puissance produite par les interactions d'individus s'imitant et s'affectant les uns les autres dans la formation de leurs désirs. Ce genre d'explication repose sur une fiction que ne cessent de reproduire les conventionnalistes tels André Orléan, qui revendiquent un individualisme méthodologique. Mais il n'est pas acceptable : les individus sont toujours déjà plongés dans une forme de vie et dans une vie sociale où le sens est institué, ce qui n'est pas tout à fait la même chose qu'être « plongés dans les interactions », comme le dit André Orléan. Ils sont sensibles à certaines valeurs et reconnaissent leur autorité parce que, à travers les usages et les institutions de leur milieu social, ils ont développé certaines pratiques qu'ils « valuent ». Cette dépendance des valeurs par rapport à des pratiques instituées « valuées » oblige à relativiser l'argument de Nathalie Heinich selon lequel les valeurs « ne reposent sur rien d'autre qu'elles-mêmes ». Ce primat des pratiques, s'il est avéré, va aussi à l'encontre du poids qu'elle donne aux « représentations axiologiques ». II oblige aussi cependant à invoquer un autre mécanisme que celui retenu par John Dewey (un type spécifique de conviction lié aux idéaux). 
Quel mécanisme ? L'explication d'André Orléan a le mérite de rapporter l'autorité des valeurs à une dynamique collective. C'est précisément celle-ci qu'il faut mieux cerner. On l'a vu, Émile Durkheim croyait à la capacité des rituels de créer des valeurs-idéaux en suscitant une effervescence collective où la vie morale est exaltée. C'est un autre type d'explication que je proposerai, en partant du constat que, dans les interactions et les actions pratiques, les individus supposent que les repères et critères qu'ils utilisent sont partagés - ceux-ci ne sont donc que putatifs ou « virtuels », dans le langage de Pierre Livet (1994). Leur partage et leur reconnaissance effectifs ne sont jamais assurés d'emblée, et seules les interactions réelles peuvent lever cette incertitude, au moins en partie. Une telle incertitude vaut en particulier pour les valeurs, du moins pour la part de celles-ci qui relève de l'engagement personnel : je ne suis jamais d'emblée assuré que les autres tiennent à ce à quoi je tiens, aient la même hiérarchie d'importance que moi, ou vénèrent ce que je vénère. Cette incertitude peut être source d'émotion, tout comme peut l'être sa réduction. En effet, le partage des émotions est souvent le moyen de s'assurer que les repères collectifs, notamment les valeurs, sont partagés et reconnus par la plupart (plutôt que par tous) comme faisant autorité pour eux. La découverte de leur non-respect par les autres peut à l'inverse susciter des affects négatifs, souvent désagréables, et faire surgir une incertitude quant à l'extension de leur partage, cette incertitude engendrant des émotions supplémentaires (Livet, 2002).

Cependant les interactions sociales disposent d'un mécanisme efficace de réduction de l'incertitude : la confiance. Comme l'a expliqué Niklas Luhmann (1968 : 24), la confiance « sert à traverser un moment d'incertitude dans le comportement des autres hommes dont on fait l'expérience au même titre que l'imprévisibilité des modifications d'un objet ». Mais il y a différents types de confiance (Quéré, 20 I7). II en est un qui joue un rôle fondamental dans la vie sociale : on peut l'appeler un Ur-trust. II s'agit d'une forme de confiance première, consistant à se fier à, à compter sur (relying on), sans hésiter, sans y penser et sans d'abord procéder à des vérifications et des évaluations. Cette confiance première est une attitude pratique, c'est-à-dire une manière d'agir. Elle est à la base de la coordination de la plupart des activités de la vie courante, où les engagements se font sans hésitation ni réflexion. Ainsi chacun se fie-t-il spontanément aux autres pour prendre comme base d'inférence et d'action les cadres institués de la vie sociale, c'est-à-dire les usages établis, les habitudes et les significations sociales, ou encore ce que tout un chacun est supposé savoir, ou tenir pour allant de soi en matière de croyances et de valeurs considérées comme légitimes concernant la vie sociale. Mais rien ne garantit qu'ils le feront effectivement, et ce sont les interactions qui servent de tests.

C'est ce que Harold Garfinkel (1967) a montré dans ses breaching experiments, en adoptant le vocabulaire de la phénoménologie sociale d'Alfred Schütz : lorsqu'ils agissent et interagissent, les agents sociaux se fient les uns aux autres pour adopter une attitude d'engagement en faveur des « croyances légitimes au sujet de la vie en société », c'est-à-dire d'allégeance aux us et coutumes, aux institutions de la vie sociale, et aux significations et valeurs instituées. Dans le vocabulaire 
phénoménologique de Harold Garfinkel, ils se fient les uns aux autres pour souscrire aux « faits naturels de la vie en société en tant que moralité », pour se référer aux « traits institutionnalisés de la collectivité » et pour le faire dans une posture tout à fait spécifique : à savoir un attachement moralement motivé, qui conjoint, comme Émile Durkheim l'a expliqué, la soumission à l'obligatoire et l'attraction de ce qui est tenu pour bon ou légitime, bref une forme de conviction morale. En d'autres termes encore, les membres « de bonne foi » de la société attendent normativement les uns des autres qu'ils se réfèrent à ce qu'ils supposent leur être commun :

«Aux structures sociales qu'ils considèrent comme faisant effectivement ou potentiellement l'objet d'une connaissance partagée avec [les autres]. Et donc, non pas à n'importe quelles structures sociales connues en commun, mais à des structures sociales normativement valuées (normatively valued social structures) que les sujets acceptent en tant que conditions que leurs jugements relativement à leur appréhension raisonnable et réaliste de leur situation, et au caractère "valable" [des points de vue des autres], doivent satisfaire. Ces structures sociales consistent en traits normatifs du système social perçu de líntérieur, qui, pour les agents, déterminent leur appartenance aux différentes collectivités auxquelles ils se réfèrent » (ibid. : 172).

Harold Garfinkel reprend ici implicitement la conception romaine des mœurs : les mœurs comme coutumes qui sont « valuées » et font autorité parce qu'elles sont supposées servir le bien commun.

Les agents sociaux, explique aussi Harold Garfinkel, appréhendent l'ordre axiologique et moral de la vie collective comme objectif, comme indépendant d'eux - une objectivité et une indépendance qu'ils produisent en fait sans s'en rendre compte. Et c'est en honorant, de manière motivée, c'est-à-dire comme une affaire de conviction morale irréfléchie, les « attentes d'arrière-plan de la vie quotidienne » qu'ils produisent les traits de la société réelle, et notamment la transcendance des normes et des valeurs. Lorsqu'un individu découvre, en cours d'interaction, que son ou ses partenaires ne se conforment pas à ces attentes, il peut être fortement désorienté, et éprouver des affects négatifs, y compris éventuellement vis-à-vis de lui-même ; il s'efforce le plus souvent de « normaliser » la situation (ibid., chap. 2).

Dans cette perspective, on peut donc dire que l'autorité des valeurs est soutenue à la fois par les attitudes que les gens adoptent dans l'accomplissement de leurs activités pratiques en interaction, et par les opérations immédiates qu'ils y font les uns par rapport aux autres : attribution hypothétique de savoirs et de croyances, supposition de partage de repères collectifs, conjecture d'un engagement commun en faveur des « faits naturels de la vie en société », ou en faveur des croyances et valeurs tenues pour légitimes, vérification implicite de ces engagements dans les interactions, etc. Fait partie de ces attitudes la « valuation » non seulement des « structures sociales 》 (dans le langage de Harold Garfinkel), mais aussi des us et coutumes, ou des pratiques instituées, en tant qu'ils sont animés par certaines valeurs et significations.

Je ne suis pas sûr que cette explication évite complètement le « sophisme du sociologue » évoqué au début. Elle est néanmoins éclairante. Ce que Harold Garfinkel appelle les « croyances légitimes au sujet de la vie en société » correspond assez bien à ce que Charles Taylor (2004) appelle un « imaginaire social ». Un 
« imaginaire « social » est une création sociale-historique ; il y a des imaginaires sociaux spécifiquement « modernes » (par exemple, l'idée d'espace public en est un, ou celle de souveraineté du peuple). II s'agit de la façon dont les gens imaginent leur existence sociale, imaginent la manière dont ils assurent leur coexistence, la façon dont les choses se passent ou devraient se passer entre eux et leurs concitoyens, etc. II ne s'agit pas seulement d'une appréhension des normes et des valeurs qui sous-tendent les interactions sociales, mais aussi d'une conception plus large de ce qui est «normal » et de ce qui ne l'est pas (la normativité sociale inclut la «normalité »), de ce qui vaut ou ne vaut pas, de ce qui est juste et ce qui ne l'est pas, ainsi que d'idées de ce qui est désirable, de ce qui devrait être, de la manière dont nous devrions vivre ensemble en société, et pour quelles raisons. Ces images de l'ordre moral de la vie en société font ainsi place à des aspirations et à des idéaux. Un imaginaire social fournit des paradigmes pour hiérarchiser, sous l'aspect de leur valeur, ou de leur mérite d'être poursuivis comme objets d'effort, les modes de comportement, les modes de vie, les manières d'interagir les uns avec les autres, les modes de gouvernement ou de gestion des affaires publiques.

S'agit-il pour autant de représentations mentales ? Nathalie Heinich (20 I 7b : 31 I) pense qu'il y a des « représentations axiologiques » de nature mentale, c'est-àdire des représentations «que se font les humains de ce qui est digne d'être apprécié ou loué, ce en quoi elles sont irréductibles à des faits de nature ». Ces représentations sont, dit-elle, culturelles et pas naturelles; elles font système et elles sont partagées. Cette caractérisation rejoint la définition ci-dessus de l'imaginaire social, mais on peut douter que ce soit en tant qu'ensemble de représentations ou de croyances que cet imaginaire guide les conduites et les pratiques, suscite des engagements et des aspirations. II me reste donc à faire un dernier pas dans la « pragmatisation » du champ de la valeur proposée par Nathalie Heinich.

\section{Conclusion}

Est indissociable de l'idée de représentation celle d'un sujet de représentations, doté d'un esprit pour loger ses représentations et faire des opérations sur elles. Nous formons bien des représentations, mais la plus grande partie de notre conduite et de nos actions repose moins sur des représentations que sur une appréhension informulée, et partiellement informulable, des choses, ou sur une appréhension tacite de notre situation d'ensemble. Cette appréhension est d'abord informulable en ceci qu'elle ne peut jamais être exprimée de manière adéquate dans des énoncés en raison de sa nature illimitée et indéfinie (Taylor, 1995).

Elle est ensuite informulable parce que ce sont les pratiques et les institutions de la vie sociale, et non pas la tête des gens, qui sont le lieu premier des significations, des normes et des valeurs. En effet, les usages, les habitudes sociales et les pratiques instituées incorporent, comme éléments constitutifs implicites, des idées, des normes, des standards et des idéaux, qui les ordonnent, les rendent praticables 
et leur donnent un sens qui suscite des engagements (voir l'exemple du vote dans un régime démocratique). C'est sous cette forme incorporée qu'opèrent d'abord les valeurs. Certes, elles peuvent être formulées, représentées, comme le sont les règles et les normes. Mais ce n'est pas d'abord comme telles qu'elles guident les conduites ; elles sont immanentes aux pratiques. C'est pourquoi, si nous sommes sensibles à certaines valeurs, c'est parce que, via des usages et des institutions, qui sont contingents, nous avons développé certaines pratiques, qui sont socialement « valuées ». Ainsi, à l'époque moderne, la conversation polie et la discussion persuasive, sous des conditions de quasi-égalité des partenaires, ont-elles remplacé aussi bien le combat ritualisé et les joutes médiévaux que les échanges entre individus appartenant à un ordre hiérarchique.

«Au commencement est l'action », écrivait Ludwig Wittgenstein dans le sillage de Goethe. II relevait ainsi que nos concepts éthiques, en particulier notre sens de la dignité humaine, sont ancrés dans ce que nous faisons, notamment dans la manière dont nous nous rapportons concrètement à notre corps, et dans la manière habituelle dont nous nous traitons mutuellement dans nos interactions. Une de ses notations des années 30 témoigne de cet ancrage:

« Mutilez complètement un homme, coupez-lui les bras et les jambes, le nez et les oreilles, et voyez alors ce qui reste de son estime de soi et de sa dignité, et jusqu'à quel point ces concepts eux-mêmes restent les mêmes. Nous ne soupçonnons pas du tout à quel point ces concepts dépendent de l'état normal, habituel de notre corps. Qu'arriverait-il si nous étions menés par une laisse attachée à un anneau passé dans notre langue? Que resterait-il d'humain en nous? Dans quel état un tel homme s'enfoncerait-il ? Nous ne savons pas que nous nous tenons sur un haut rocher étroit entouré de précipices, dans lesquels tout semble différent ${ }^{8} \gg$ (cité in Shusterman, 2002 : 103- 104 ).

Si nous transposons cette notation à la question des valeurs, on peut dire que celles-ci ne sont pas séparées d'un « état normal, habituel », socialement « valué », des interactions et des relations sociales, et plus largement des modes de coexistence. Cet état peut aussi être éventuellement critiqué pour ses défauts et ses manques, au regard de possibilités réelles révélées par l'expérience - le « normal » se conjugue en effet non seulement à l'indicatif, mais aussi au conditionnel (« ce qui serait normal » ce serait de... ou que... ). Mais il est surtout contingent... et « entouré de précipices »!

\section{Références}

Bouveresse J., 2006, « Combien mesure le mètre ? § 50 », pp. 4I-84, in : Laugier S., Chauviré C., éds, Lire les Recherches philosophiques de Wittgenstein, Paris, Vrin.

Chauviré C., 2004, Le Moment anthropologique de Wittgenstein, Paris, Kimé.

\footnotetext{
${ }^{8}$ " Mutilate completely a man, cut off his arms and legs, nose and ears, and then see what it remains of his self-respect and his dignity, and to what point his concepts of these things are still the same. We don't suspect at all, how these concepts depend on the habitual, normal state of our bodies. What would happen to them if we were led by a leash attached to a ring through our tongues? How much then still remains of a man in him? Into what state does such a man sink? We don't know that we are standing on a high narrow rock and surrounded by precipices, in which everything looks different ».
} 
Dewey J., 1925, Expérience et nature, trad. de l'anglais par J. Zask, Paris, Gallimard, 2012.

Dewey J., 1930, « Conduct and experience », pp. 409-422, in: Murchison C., ed., Psychologies of 1930, Worcester, Clark University Press.

Dewey J., 1931, « Qualitative Thought », pp. 93- I 16, in: Dewey J., Philosophy and Civilization, New York, Minton, Balch \& Company.

Dewey J., 1934a, Fuvres philosophiques. T. III. L'Art comme expérience, trad. de l'anglais par J.-P. Cometti et al., Pau, Publications de l'Université de Pau, 2005.

Dewey J., 1934b, Une foi commune, trad. de l'anglais par P. Di Masio, Paris, Éd. Les Empêcheurs de penser en rond, $201 \mathrm{I}$.

Dewey J., 1938, La Formation des valeurs, Paris, Éd. Les Empêcheurs de penser en rond, 201 I.

Dewey J., 1939, « Experience, knowledge and value: a rejoinder », pp. 517-608, in: Schilpp P.A., ed., The Philosophy of John Dewey, Evanston, Northwestern University (la pagination des citations est celle des Later Works, vol. I4, pp. 3-76).

Dewey J., 1949, «The field of "value" », pp. 64-77, in: Lepley R., Value: a Cooperative Inquiry, New York, Columbia University Press (la pagination des citations est celle des Later Works, vol. 16, pp. 343-357).

Durkheim É., 1912, Les Formes élémentaires de la vie religieuse. Le système totémique en Australie, Paris, Presses universitaires de France, 2008.

Durkheim É., 1970, La Science sociale et l'action, Paris, Presses universitaires de France.

Garfinkel H., 1967, Recherches en ethnométhodologie, trad. de l'anglais par M. Barthélémy et al., Paris, Presses universitaires de France, 2007.

Habermas J., 1992, Droit et démocratie, trad. de l'allemand par R. Rochlitz, C. Bouchindhomme, Paris, Gallimard, 1997.

Heinich N., 2017a, Des valeurs. Une approche sociologique, Paris, Gallimard.

Heinich N., 20 I7b, « Dix propositions sur les valeurs », Questions de communication, 31, pp. 291-313.

Hochschild A. R., 1979, « Emotion work, feeling rules and social structure », American Journal of Sociology, 85, 3, pp. 55I-575.

James W., 1909, Philosophie de l'expérience, trad. de l'anglais par S. Galetic, Paris, Éd. Les Empêcheurs de penser en rond, 2007.

Joas H., 20 I3, The Sacredness of the Person. A New Genealogy of Human Rights. Washington, Georgetown University Press.

Livet P., 1994, La Communauté virtuelle. Action et communication, Combas, Éd. de L'Éclat.

Livet P., 2002, Émotions et rationalité morale, Paris, Presses universitaires de France.

Luhmann N., 1968, La Confiance. Un mécanisme de réduction de la complexité sociale, trad. de l'allemand par S. Bouchard, Paris, Éd. Economica, 2006.

Mclntyre A., 1981, After Virtue, Notre Dame, University of Notre Dame Press, 1984.

Orléan A., 201 I, L'Empire de la valeur. Refonder l'économie, Paris, Éd. Le Seuil.

Parsons T., 1968, « On the concept of value-commitments », Sociological Inquiry, 38, 2, pp. I35- 160. 
Quéré L., 20 17, « La confiance sans le savoir. De Simmel à Dewey et Wittgenstein », pp. 249265, in :Thouard D., Zimmermann B., éds, Georg Simmel. Différenciation et réciprocité, Paris, Éd. CNRS.

Shusterman R., 2002, «Wittgenstein's somaesthetics: body feeling in philosophy of mind, art and ethics », Revue internationale de philosophie, 219, pp. 91- 108.

Taylor C., 1989, Les Sources du moi. La formation de l'identité moderne, trad. de l'anglais par C. Melançon, Paris, Éd. Le Seuil, 1998.

Taylor C., 1995, « Suivre une règle », trad. de l'anglais par C. Fournier et C. Chauviré, Critique, 579-580, pp. 554-572.

Taylor C., 2004, Modern Social Imaginaries, Durham, Duke University Press. 